\title{
Validité des diagnostics d'autisme recensés à l'aide de données administratives sur la santé
}

L. Dodds, Ph.D. (1); A. Spencer, M.Sc. (1); S. Shea, M.D. (2);D. Fell, M.Sc. (1); B.A. Armson, M.D. (3) A.C. Allen, M.D. (1);

S. Bryson, Ph.D. (2)

\section{Résumé}

Il est nécessaire de surveiller la prévalence de l'autisme afin de planifier l'aide à l'éducation et les services de santé à offrir aux enfants qui en sont atteints. C'est pourquoi nous avons réalisé cette étude qui vise à évaluer l'exactitude des bases de données administratives sur la santé en ce qui concerne les diagnostics d'autisme. Pour ce faire, nous avons repéré les diagnostics de troubles du spectre autistique (TSA) dans trois bases de données administratives sur la santé de la province de la Nouvelle-Écosse, soit la base de données sur les congés d'hôpital, la base de données sur la facturation des médecins au régime d'assurance-santé et la base de données du Système d'information sur les patients en clinique externe de santé mentale. Nous avons construit sept algorithmes en variant le nombre requis de déclarations de TSA (une ou plusieurs) et le nombre de bases de données administratives (une à trois) dans lesquelles ces déclarations doivent se retrouver. Nous avons ensuite comparé, avec chaque algorithme, les diagnostics posés par l'équipe attitrée à l'autisme du Centre de santé Izaak Walton Killam (IWK) à l'aide des barèmes de diagnostic de l'autisme les plus perfectionnés, puis nous avons calculé la sensibilité, la spécificité et la statistique C (une mesure de la capacité discriminante du modèle). L’algorithme présentant les meilleures caractéristiques d'essai était celui qui exigeait un code de TSA présent dans n'importe laquelle des trois bases de données (sensibilité = $69,3 \%$ ). La sensibilité de l'algorithme fondé sur la présence d'au moins un code de TSA soit dans la base de données sur les congés d'hôpital soit dans la base de données sur la facturation des médecins, était de 62,5 \%. Les bases de données administratives sur la santé pourraient représenter une source économique pour la surveillance de l'autisme, surtout par comparaison aux méthodes nécessitant la collecte de nouvelles données. Cependant, il faudrait des sources de données additionnelles pour améliorer la sensibilité et l'exactitude des méthodes de recensement des cas d'autisme au Canada.

\section{Introduction}

Selon les données disponibles, la prévalence des troubles du spectre autistique (TSA) en général, et de l'autisme, en particulier, augmenterait avec le temps ${ }^{1-4}$. Si cette affirmation s'avère exacte, d'importantes conséquences sont à prévoir sur le système d'éducation et les organismes qui fournissent des services pour ces enfants : l'offre d'aide et de services ne suffira pas à répondre aux demandes sans cesse croissantes adressées au système d'éducation et aux fournisseurs de services de santé. Jusqu'ici, des projets isolés au Canada ont permis d'estimer la prévalence des TSA dans certains territoires, mais il n'y a actuellement aucun système en place pour suivre régulièrement l'incidence et la prévalence de l'autisme et en rendre compte. La surveillance active de l'autisme, qui s'effectue par le dépistage dans la population, fournit d'excellents renseignements sur la prévalence, mais cette méthode est coûteuse et n'est généralement appliquée qu'à des enquêtes à court terme ${ }^{1}$. La surveillance passive de l'autisme, qui s'appuie sur les bases de données existantes, constitue une méthode relativement économique grâce à laquelle il est possible d'obtenir une estimation continue de la prévalence dans la population.

Le vaste éventail des troubles cognitifs et neurocomportementaux connexes, dont l'autisme est le trouble le plus grave, est ce qu'on appelle les troubles envahissants du développement (TED) ou les troubles du spectre autistique (TSA) $)^{1,5}$. Selon les critères de diagnostic de la Classification internationale des maladies (CIM-10) établie par l'Organisation mondiale de la santé (OMS), les TED englobent l'autisme infantile, l'autisme atypique, le syndrome de Rett, les autres troubles désintégratifs de l'enfance, l'hyperactivité associée à un retard mental et à des mouvements stéréotypés, le syndrome d'Asperger, les autres troubles envahissants du développement et les troubles envahissants du développement sans précision. L'autisme infantile, l'autisme atypique et le syndrome d'Asperger sont les diagnostics les plus courants. Dans le présent article, nous utiliserons le terme TSA, qui regroupe tous les TED sauf le syndrome de Rett et les troubles désintégratifs de l'enfance, lesquels sont extrêmement rares.

En 1985, Bryson et al. ont tenté pour la première fois d'estimer la prévalence de l'autisme au Canada en effectuant un dépistage chez tous les enfants

Coordonnées des auteurs

1 Unité de recherche en épidémiologie périnatale, Département d’obstétrique et de gynécologie et Département de pédiatrie, Université Dalhousie, Halifax (Nouvelle-Écosse) 2 Département de pédiatrie, Université Dalhousie, Halifax (Nouvelle-Écosse)

3 Département d'obstétrique et de gynécologie, Université Dalhousie (Nouvelle-Écosse)

Correspondance : Linda Dodds, Ph.D., Département d'obstétrique et de gynécologie et Département de pédiatrie, Université Dalhousie, 5850-5980, avenue University, C.P. 9700, Halifax (Nouvelle-Écosse) B3K 6R8, Tél. : 902-470-7191; Téléc. : 902-470-7190; Courriel : I.dodds@dal.ca 
de 6 à 14 ans ( $n=20$ 800) vivant dans une zone géographique donnée de la Nouvelle-Écosse, et en réalisant des évaluations diagnostiques de suivi chez les enfants qui se sont avérés positifs au dépistage $(n=46)^{6}$. Des 46 cas positifs, 21 cadraient dans le spectre relativement étroit de l'autisme tel qu'il était défini à ce moment-là (la majorité d'entre eux, si ce n'est la totalité, répondraient aux critères les plus rigoureux des troubles autistiques) ${ }^{7}$.

Plus récemment, des chercheurs canadiens ont utilisé les données existantes pour estimer la prévalence des TSA. Ouellette-Kuntz et al. ont estimé la prévalence des TED chez les enfants de 15 ans ou moins pour l'année 2002 dans les provinces de l'Île-du-Prince-Édouard et du Manitoba ${ }^{8}$. À l'île-du-Prince-Édouard, les cas ont été relevés par le ministère des Services sociaux et des Aînés et le ministère de l'Éducation. Les chercheurs ont dû préalablement obtenir le consentement des parents pour recueillir les renseignements. Au Manitoba, les cas ont été repérés à partir des orientations vers le programme des services spéciaux pour enfants du ministère des Services à la famille et du Logement. Les taux de prévalence des TED chez les enfants de 1 à 15 ans dans les deux provinces se sont révélés semblables (soit 2,84 cas pour 1000 , au Manitoba, et 3,52 pour 1000 , à l'îledu-Prince-Édouard). Fombonne et al. ont mesuré la prévalence des TED dans une population d'enfants inscrits dans une grande commission scolaire anglophone de la région de Montréal au $1^{\text {er }}$ octobre 2003 $\left(\mathrm{n}=27\right.$ 749) ${ }^{9}$. Au Québec, les commissions scolaires fournissent au ministère de l'Éducation des renseignements sur les enfants présentant des TED ou d'autres troubles afin d'obtenir un financement supplémentaire. Dans le cadre de cette enquête menée en 2003, en tout 180 enfants ont reçu le diagnostic de TED (6,5 enfants pour 1 000), et 61 d'entre eux ont reçu le diagnostic plus précis d'autisme ${ }^{9}$. En résumé, la surveillance et le signalement de cas d'autisme au Canada sont peu fréquents, et les taux sont variables.

Jusqu'ici, aucune base de données administratives sur la santé n'a été utilisée au Canada pour estimer l'incidence ou la prévalence de l'autisme, bien qu'elles aient servi à évaluer l'incidence ou la prévalence d'autres problèmes de santé (p. ex. des algorithmes ont été construits et mis à l'essai à partir de données administratives pour déterminer l'incidence et la prévalence de l'asthme infantile, de l'ostéoporose, du diabète de type 2 et de l'œdème maculaire dû au diabète $\left.{ }^{10-13}\right)$. Dans une étude visant à évaluer la validité des codes de la CIM figurant dans les données administratives sur les congés d'hôpital, Quan et al. ${ }^{14}$ ont comparé les codes CIM-9 et CIM-10 (c.-à-d. ceux utilisés dans les bases de données administratives sur la santé) aux données inscrites dans les dossiers médicaux, en considérant 32 problèmes de santé autres que les TSA. Les chercheurs ont constaté que le taux de détection (c.-à-d. la sensibilité) variait selon le problème de santé : de $82 \%$, pour l'insuffisance rénale, à $9 \%$, pour la perte de poids ${ }^{14}$.

Les bases de données administratives sur la santé sont une source qui pourrait permettre de mesurer la prévalence de l'autisme dans une population donnée, à condition que la validité des diagnostics de TSA tirés des données administratives sur la santé puisse être établie auparavant. À l'aide d'une étude de cohorte d'enfants nés en Nouvelle-Écosse entre 1989 et 2002, nous avons apparié des bases de données administratives sur la santé à une base de données cliniques de référence sur l'autisme pour évaluer l'exactitude des diagnostics d'autisme recensés à l'aide des bases de données administratives sur la santé.

\section{Méthode}

La présente étude s'appuie sur des données issues d'une étude de cohorte rétrospective visant à examiner les facteurs prénataux, obstétriques et néonataux liés à l'apparition de l'autisme. Nous avons constitué une cohorte en recensant tous les enfants nés en Nouvelle-Écosse entre 1989 et 2002 inscrits dans la base de données périnatales Atlee (Atlee Perinatal Database), une base de données populationnelle sur tous les enfants nés en milieu hospitalier en NouvelleÉcosse. Ensuite, nous avons apparié cette cohorte d'enfants aux bases de données administratives sur la santé de l'Unité de recherche sur la santé de la population de l'Université Dalhousie, puis nous avons établi la correspondance des données à l'aide des numéros de carte santé codés qui figurent dans toutes les sources de données. Les bases de données administratives sur la santé nous ont permis de suivre ces enfants jusqu'en décembre 2005.

Pour les résidants de la Nouvelle-Écosse, comme pour tous les Canadiens, l'accès aux services hospitaliers ou médicaux est universel et fourni par l'intermédiaire d'un régime public de soins de santé. Dans cette étude, nous avons repéré les diagnostics de TSA dans trois bases de données administratives sur la santé de la Nouvelle-Écosse, soit la base de données sur les congés d’hôpital (créée en 1989), la base de données sur la facturation des médecins au régime d'assurance-santé (créée en 1989) et la base de données du Système d'information sur les patients en clinique externe de santé mentale (créée en 1992). La base de données sur les congés d'hôpital contient les diagnostics qui sont consignés dans les dossiers médicaux des patients et résumés au moment du congé d'hôpital. La base de données sur la facturation des médecins au régime d'assurance-santé comprend un ou plusieurs codes de diagnostic consignés par un médecin et transmis à l'organisme provincial de paiement des services assurés. La base de données du Système d'information sur les patients en clinique externe de santé mentale réunit des données sur tous les patients reçus dans les cliniques externes de santé mentale et ceux suivant un programme de traitement de jour de santé mentale. Les diagnostics qui y figurent ont été consignés par un psychiatre ou un psychologue, ou les deux. Dans ces trois bases de données administratives, le diagnostic de TSA a été défini comme l'inscription du code 299 (CIM-9) ou du code F84 (CIM-10) dans le champ du diagnostic primaire ou secondaire.

Nous avons construit sept algorithmes en variant le nombre requis de déclarations de TSA (une ou plusieurs) devant se trouver dans les trois bases de données administratives. Par exemple, dans un algorithme, un enfant est considéré comme ayant reçu un diagnostic d'autisme si au moins un code d'autisme lui a été attribué 
TABLEAU 1

Résultats des différents algorithmes ${ }^{1}$ exigeant un certain nombre de diagnostics de troubles du spectre de l'autisme (TSA) dans les trois bases de données administratives sur la santé, en fonction des diagnostics de référence

\begin{tabular}{|c|c|c|c|c|c|c|c|c|c|}
\hline \multicolumn{3}{|c|}{ Type de données administratives } & \multicolumn{4}{|c|}{ Résultats comparés au diagnostic de référence } & \multicolumn{3}{|c|}{ Caractéristiques des algorithmes } \\
\hline $\begin{array}{l}\text { Données sur } \\
\text { les congés } \\
\text { d'hôpital } \\
\text { (nombre de } \\
\text { codes de TSA) }\end{array}$ & $\begin{array}{l}\text { Données } \\
\text { sur la } \\
\text { facturation } \\
\text { des médecins } \\
\text { (nombre } \\
\text { de codes } \\
\text { de TSA) }\end{array}$ & $\begin{array}{l}\text { Données sur } \\
\text { les patients } \\
\text { en clinique } \\
\text { externe de } \\
\text { santé mentale } \\
\text { (nombre de } \\
\text { codes de TSA) }\end{array}$ & $\begin{array}{c}\text { Nombre de } \\
\text { vrais positifs }\end{array}$ & $\begin{array}{l}\text { Nombre de } \\
\text { vrais négatifs }\end{array}$ & $\begin{array}{l}\text { Nombre de } \\
\text { faux positifs }\end{array}$ & $\begin{array}{l}\text { Nombre de } \\
\text { faux négatifs }\end{array}$ & Sensibilité & Spécificité & Statistique C \\
\hline \multirow[t]{3}{*}{$\geq 1$} & & & 21 & 86 & 2 & 155 & $11,9 \%$ & $97,7 \%$ & 0,55 \\
\hline & $\geq 1$ & & 105 & 75 & 13 & 71 & $59,7 \%$ & $85,2 \%$ & 0,72 \\
\hline & & $\geq 1$ & 29 & 81 & 7 & 147 & $16,5 \%$ & $92,0 \%$ & 0,54 \\
\hline$\geq 1$ & $\geq 1$ & $\geq 1$ & 122 & 68 & 20 & 54 & $69,3 \%$ & $77,3 \%$ & 0,76 \\
\hline$\geq 1$ & $\geq 1$ & & 110 & 73 & 15 & 66 & $62,5 \%$ & $83,0 \%$ & 0,74 \\
\hline$\geq 1$ & $\geq 2$ & $\geq 2$ & 75 & 78 & 10 & 101 & $42,6 \%$ & $88,6 \%$ & 0,67 \\
\hline$\geq 1$ & $\geq 2$ & & 65 & 82 & 6 & 111 & $36,9 \%$ & $93,2 \%$ & 0,65 \\
\hline
\end{tabular}

${ }^{1}$ Lorsque les algorithmes sont basés sur des codes d'autisme de plus d'une base de données, un diagnostic d'autisme a été conféré si un code d'autisme a été utilisé dans une des bases de données en question.

dans la base de données sur les congés d'hôpital; la présence d'un tel code dans les autres bases de données n'est pas nécessaire. L'algorithme permettant d'obtenir le plus grand nombre de diagnostics d'autisme exigeait la présence d'au moins une déclaration de TSA dans l'une des trois bases de données énumérées plus haut.

Les diagnostics de référence ont été tirés d'une base de données cliniques créée par l'équipe attitrée à l'autisme du Centre de santé IWK. Cette équipe s'était chargée d'évaluer des cas d'autisme soupçonnés qui lui avaient été adressés en grande partie par des professionnels de la santé et par quelques enseignants de la municipalité régionale de Halifax. L'équipe était composée de pédiatres, de psychologues, de travailleurs sociaux, de psychiatres, d'orthophonistes, d'ergothérapeutes et de membres $\mathrm{du}$ personnel infirmier. Les diagnostics finals ont été posés par les psychologues et/ou les pédiatres ou les psychiatres qui ont dirigé ou co-dirigé les équipes de diagnostic; les diagnostics reposaient sur l'entrevue ADI-révisée (Autism Diagnostic Interview-revised), l'échelle ADOS (Autism Diagnostic Observation Schedule) et le jugement clinique s'appuyant sur le DSM-IV-TR ${ }^{15-17}$. Ces instruments et ces critères sont conformes aux paramètres recommandés de la pratique pour le diagnostic des $\mathrm{TSA}^{18,19}$. Les diagnostics posés par l'équipe, considérés comme les diagnostics de référence, ont été consignés dans une base de données à partir de 2001.

Dans l'établissement de correspondances entre la base de données périnatales Atlee, les bases de données administratives sur la santé et la base de données de référence, nous avons veillé à protéger l'anonymat en suivant une procédure à plusieurs étapes. La première étape consistait à créer un fichier de concordance dans lequel un numéro unique était assigné à chaque patient dans chacune des bases de données et jumelé au numéro de carte santé codé de ces patients. Une tierce partie a utilisé un algorithme sophistiqué pour encoder le numéro de carte santé que possède chaque citoyen de la province, ainsi qu'un champ commun à toutes les sources de données. Finalement, les variables demandées dans chaque dossier ont été reliées à nouveau au fichier de concordance (à l'aide du numéro codé propre à chaque patient et qui figure dans chaque base de données), et un fichier d'analyse anonyme correspondant, renfermant les éléments tirés de chaque source de données, a été créé.

Nous avons comparé les diagnostics posés de 2001 à 2005 par l'équipe du Centre de santé IWK (les données de référence) aux diagnostics de TSA établis à l'aide de chacun des sept algorithmes construits à partir des bases de données administratives sur la santé. Nous avons évalué l'exactitude des résultats de chaque algorithme en calculant la sensibilité, la spécificité et la statistique C (une estimation non paramétrique de l'aire sous la courbe de la fonction d'efficacité du récepteur, ou Receiver Operating Characteristic Curve, qui donne une indication sur la capacité d'une méthode de prévoir un diagnostic d'autisme). Les scores de la statistique $\mathrm{C}$ varient de 1,0 , pour un algorithme " parfait » (dont la sensibilité et la spécificité étaient de $100 \%$ ), à 0,5, pour un algorithme non discriminant ${ }^{20}$.

Pour repérer les vrais cas de TSA qui n'ont pas été relevés dans les bases de données administratives (p. ex. les faux négatifs), nous avons considéré les codes désignant d'autres problèmes de santé psychologiques. De plus, nous avons examiné les codes qui ont été consignés à la fois avant et après la date du vrai diagnostic (c.-à-d. le diagnostic de référence). Dans le cas des patients à qui un code d'autisme avait été attribué dans l'une des bases de données administratives, mais qui, après évaluation par l'équipe de diagnostic, ne s'avéraient pas atteints de TSA (c.-à-d. les faux positifs), nous avons voulu connaître le nombre de déclarations incorrectes et les années où elles ont été inscrites, déterminer si elles avaient été consignées après la date du diagnostic négatif par l'équipe de diagnostic du Centre 
TABLEAU 2

Comparaison du nombre de déclarations de troubles du spectre de l'autisme (TSA) par enfant, entre les faux positifs et les vrais positifs

\begin{tabular}{|c|c|c|c|}
\hline & & Faux positifs & Vrais positifs \\
\hline Base de données & $\begin{array}{c}\text { Nombre de } \\
\text { déclarations de } \\
\text { TSA par enfant }\end{array}$ & $\begin{array}{l}\text { Nombre d'enfants (\%) } \\
\qquad(\mathrm{n}=\mathbf{2 0})\end{array}$ & $\begin{array}{l}\text { Nombre d'enfants (\%) } \\
\qquad(n=122)\end{array}$ \\
\hline \multirow[t]{2}{*}{ Congés d'hôpital } & Au moins 1 & $2(10 \%)$ & $21(17 \%)$ \\
\hline & Deux ou plus & $1(5 \%)$ & $7(6 \%)$ \\
\hline \multirow{2}{*}{$\begin{array}{l}\text { Facturation des } \\
\text { médecins au régime } \\
\text { d'assurance-santé }\end{array}$} & Au moins 1 & $13(65 \%)$ & $104(85 \%)$ \\
\hline & Deux ou plus & $4(20 \%)$ & $55(45 \%)$ \\
\hline \multirow{2}{*}{$\begin{array}{l}\text { Système d'information } \\
\text { sur les patients en } \\
\text { clinique externe de } \\
\text { santé mentale }\end{array}$} & Au moins 1 & $7(35 \%)$ & $29(24 \%)$ \\
\hline & Deux ou plus & $7(35 \%)$ & $23(19 \%)$ \\
\hline \multirow{2}{*}{$\begin{array}{l}\text { Présence dans l'une des } \\
\text { trois bases de données }\end{array}$} & Au moins 1 & $20(100 \%)$ & $122(100 \%)$ \\
\hline & Deux ou plus & $11(55 \%)$ & $74(61 \%)$ \\
\hline
\end{tabular}

TABLEAU 3

Sensibilité et spécificité des diagnostics de troubles du spectre de l'autisme (TSA) recensés à l'aide de données administratives par rapport aux diagnostics de référence, en fonction de facteurs liés à la mère et à la néonatalité

\begin{tabular}{|c|c|c|c|}
\hline Facteur & Nombre & Sensibilité (IC à 95 \%) & Spécificité (IC à $95 \%$ ) \\
\hline \multicolumn{4}{|l|}{ Âge de la mère } \\
\hline$<35$ & 218 & $71,2 \%$ (63,2 à 78,1) & $77,2 \%(66,8$ à 85,2$)$ \\
\hline$\geq 35$ & 46 & $62,2 \%(46,1$ à 76,0$)$ & 77,8 \% (44,3 à 94,7) \\
\hline \multicolumn{4}{|l|}{ Lieu de résidence } \\
\hline Comté de Halifax & 214 & $68,1 \%(60,0$ à 75,1$)$ & $80,0 \%(69,1$ à 87,8$)$ \\
\hline Autre & 50 & 75,0 \% (57,7 à 87,0) & 66,7 \% (43,6 à 83,9) \\
\hline \multicolumn{4}{|l|}{ Poids à la naissance } \\
\hline$<2500 \mathrm{~g}$ & 15 & 72,7 \% (42,9 à 90,8) & 75,0 \% (28,9 à 96,6) \\
\hline$\geq 2500 \mathrm{~g}$ & 234 & 69,1 \% (61,7 à 75,7) & 77,4 \% (67,3 à 85,1) \\
\hline \multicolumn{4}{|c|}{ Présence d'une anomalie congénitale importante ${ }^{*}$} \\
\hline Oui & 13 & 55,6 \% (26,6 à 81,2) & 100 \% (45,4 à 100) \\
\hline Non & 247 & $69,9 \%(62,5$ à 76,4) & 76,5 \% (66,2 à 84,5) \\
\hline \multicolumn{4}{|l|}{ Sexe } \\
\hline Garçon & 231 & 69,7 \% (62,0 à 76,4) & 80,3 \% (69,8 à 87,8) \\
\hline Fille & 33 & 66,7 \% (45,2 à 83,0) & 58,3 \% (31,9 à 80,7) \\
\hline \multicolumn{4}{|l|}{ Rang de naissance } \\
\hline Aîné & 145 & $69,8 \%$ (60,0 à 78,1) & $77,6 \%$ (64,0 à 87,1) \\
\hline Cadet & 119 & 68,8 \% (57,9 à 77,9) & 76,9 \% (61,5 à 87,6) \\
\hline
\end{tabular}

* Une anomalie importante est définie comme un défaut de structure ou de fonction qui est présent à la naissance et qui affecte la longévité ou la qualité de vie, ou qui requiert une chirurgie. de santé IWK et savoir s'il y avait eu d'autres déclarations relatives à des problèmes psychologiques. Nous avons comparé la sensibilité et la spécificité des diagnostics en fonction de facteurs relatifs à la mère ou à son nourrisson, comme le petit poids à la naissance et l'âge de la mère (données de la base de données périnatales Atlee), pour savoir si certaines caractéristiques étaient associées à l'exactitude des diagnostics d'autisme tirés des données administratives sur la santé.

Cette étude a été approuvée par le comité d'éthique pour la recherche du Centre de santé IWK.

\section{Résultats}

L'équipe de diagnostic a évalué 270 patients de la cohorte générale des enfants nés en Nouvelle-Écosse visée par notre étude. Les résultats étaient les suivants : 176 cas ont reçu une confirmation du diagnostic de TSA et 88 cas n'étaient pas des cas de TSA (6 patients ont obtenu un diagnostic indéterminé et ont donc été rejetés aux fins des analyses ultérieures). Il y avait pour les 264 enfants qui restent des données administratives échelonnées sur au moins 2 ans suivant leur date de naissance. Au moment de l'évaluation faite par l'équipe de diagnostic, $58 \%$ des enfants avaient 4 ans ou moins; seulement $12 \%$ des enfants avaient 10 ans ou plus. Dans la majorité des cas confirmés, le code attribué correspondait à un diagnostic général de TSA sans mention d'un diagnostic précis d'autisme.

Le tableau 1 présente la définition de cas de chacun des sept algorithmes mis à l'essai ainsi que la sensibilité, la spécificité et la statistique C qui leur sont propres. L'algorithme ayant obtenu les valeurs les plus élevées pour la statistique C (soit 0,76 ) et la sensibilité (soit 69,3\%), et une spécificité de $77,3 \%$, est celui qui permettait d'établir un diagnostic de TSA par la présence d'au moins une déclaration dans l'une des trois bases de données administratives. Avec cet algorithme, le diagnostic établi était correct chez 190 des 264 enfants. Parmi les mauvais diagnostics, il y a eu 20 faux positifs et 54 faux négatifs, que nous avons examinés de plus près pour 
nous aider à expliquer les erreurs constatées dans les bases de données administratives.

Nous nous sommes penchés sur les 54 faux négatifs, c.-à-d. les enfants qui ont reçu le diagnostic de TSA après évaluation par l'équipe de diagnostic mais pour qui aucune déclaration de TSA n’a été relevée dans les trois bases de données, pour vérifier si d'autres déclarations pouvaient avoir été systématiquement consignées à la place d'un diagnostic de TSA. Des 54 faux négatifs, 46 se sont vu attribuer au moins une déclaration dans la base de données sur la facturation des médecins au régime d'assurance-santé; ces mentions étaient relatives à des troubles névrotiques, à des troubles de la personnalité ou à d'autres troubles mentaux non psychotiques (codes 300 à 316 de la CIM-9). De ces 46 faux négatifs, 35 (soit $76 \%$ ) se sont vu attribuer au moins une fois le code 315 de la CIM-9 (" retards spécifiques du développement»). Ce code a été relevé pour 22 enfants avant la date du diagnostic posé par l'équipe, et pour 26 enfants, après cette date; pour un certain nombre d'enfants, le code a été donné à la fois avant et après cette date.

Pour les 20 faux positifs et les 122 vrais positifs (voir le tableau 2), nous avons comparé le nombre de déclarations de TSA inscrites par enfant dans chacune des trois bases de données. Parmi les 20 faux positifs, le code de TSA a été attribué à 2 enfants (soit $10 \%$ ) dans la base de données sur les congés d'hôpital, à 13 enfants (soit $65 \%$ ) dans celle sur la facturation des médecins au régime d'assurance-santé et à 7 enfants (soit $35 \%$ ) dans celle du Système d'information sur les patients en clinique externe de santé mentale (voir le tableau 2). Des 13 enfants du groupe des faux positifs auxquels au moins une déclaration de TSA avait été attribuée dans la base de données sur la facturation des médecins au régime d'assurance-santé, 4 (soit $31 \%$ ) se sont vu attribuer plus d'une déclaration de TSA dans cette base de données, par comparaison à 55 dans le groupe des 104 vrais positifs (soit $53 \%$ ). Dans le groupe des faux positifs relevés dans le Système d'information sur les patients en clinique externe de santé mentale, tous les patients s'étaient vu attribuer plus d'une déclaration de TSA. Des 122 vrais positifs,
21 cas (soit $17 \%$ ) avaient au moins une déclaration dans la base de données sur les congés d'hôpital, 104 cas (soit 85 \%), dans la base de données sur la facturation des médecins au régime d'assurance-santé et 29 cas (soit 24 \%), dans le Système d'information sur les patients en clinique externe de santé mentale. Pour 27 enfants (soit $22 \%$ ), on trouvait une déclaration de TSA dans deux bases de données et pour 5 enfants (soit $4 \%$ ), on en trouvait dans l'ensemble des trois bases (données non présentées). S'il est vrai que la plupart des déclarations de TSA figurant dans la base de données sur les congés d'hôpital et celle du Système d'information sur les patients en clinique externe de santé mentale ont été inscrites après le diagnostic de vrai positif posé par l'équipe de diagnostic, 55 des 104 enfants (soit 53 \%) se sont vu attribuer au moins une déclaration dans la base de données sur la facturation des médecins au régime d'assurance-santé avant le diagnostic. Mis à part les codes de TSA, le code le plus fréquent a été le 315 de la CIM-9-MC (" retards spécifiques du développement »); ce code a été attribué autant de fois avant qu'après la date du diagnostic posé par l'équipe.

Nous avons comparé la sensibilité et la spécificité des diagnostics en fonction de caractéristiques propres à la mère et à la néonatalité (voir le tableau 3). La sensibilité des données administratives pour révéler un diagnostic de TSA était semblable d'un facteur à l'autre dans la majorité des cas, y compris entre les garçons $(69,7 \%)$ et les filles $(66,7 \%)$. La sensibilité des données administratives à révéler un diagnostic de TSA n'a pas été significativement plus faible chez les enfants présentant une anomalie congénitale importante $(55,6 \%)$ que chez les enfants sans anomalie $(69,9 \%)$. La sensibilité n’a pas été significativement plus élevée chez les enfants vivant à l'extérieur du comté de Halifax par rapport à ceux qui y résident $(75,0 \%$ par comparaison à $68,1 \%)$, bien que la spécificité soit plus faible $(66,7 \%$ par comparaison à $80,0 \%$, respectivement).

\section{Analyse}

Dans la présente étude, nous avons utilisé les codes inscrits dans trois bases de données administratives sur la santé pour évaluer l'efficacité de divers algorithmes à repérer les cas d'autisme parmi les enfants de la Nouvelle-Écosse. Bien que la cohorte de l'étude englobe tous les enfants nés en Nouvelle-Écosse entre 1989 et 2002, seuls les enfants de cette cohorte rencontrés par l'équipe de diagnostic entre 2001 et 2005 ont été admis dans cette étude de validation. D'après l'algorithme définissant les cas d'autisme par la présence d'au moins une déclaration dans l'une des trois bases de données (sur les congés d'hôpital, sur la facturation des médecins au régime d'assurance-santé ou sur les patients en clinique externe de santé mentale), la capacité des bases de données administratives sur la santé de la NouvelleÉcosse à répertorier correctement les cas d'autisme s'est avérée modérée (sensibilité de $69 \%$ ). Dans la plupart des cas où les TSA ont été mal diagnostiqués dans les bases de données administratives (c.-à-d. les faux négatifs), des codes indiquant quelque autre trouble psychologique non psychotique ou un retard de développement ont été consignés, ce qui semble indiquer que les médecins ont peut-être été réticents à attribuer un code d'autisme avant la confirmation d'un diagnostic d'autisme.

L'une des forces de cette étude a été la qualité du diagnostic d'autisme dans la population de référence. Cependant, le diagnostic de référence n'a été posé que chez les enfants qui ont été orientés vers l'équipe de diagnostic de l'autisme. Il est à noter que, dans cette étude de validation, les enfants sans diagnostic de TSA au moment de l'évaluation par l'équipe auraient présenté dans leur comportement et/ou leur développement certaines caractéristiques qui auraient justifié une orientation vers l'équipe. Par conséquent, le taux de faux positifs obtenu dans cette étude est probablement plus élevé, et la spécificité, plus faible, qu'ils ne l'auraient été si nous avions pu établir un diagnostic de référence pour tous les enfants figurant dans les bases de données administratives. Néanmoins, la spécificité que nous avons calculée est raisonnablement élevée (soit 
$77 \%$ ), une estimation probablement inférieure à la spécificité réelle. Dans les autres algorithmes utilisés dans cette étude, les exigences étaient plus rigoureuses pour la définition de cas d'autisme (p. ex. deux déclarations de médecins étaient requises), ce qui améliorait la spécificité (par rapport à celle de l'algorithme qui n'exigeait qu'une déclaration) aux dépens de la sensibilité.

Afin d'améliorer le taux de détection obtenu dans cette étude, il faudrait utiliser d'autres sources de données. Au Canada, les renseignements sur les diagnostics de TSA sont disponibles dans les conseils scolaires de certaines régions ou dans certains ministères provinciaux responsables des services sociaux ou des services à la famille, tel que mentionné plus haut. Aux États-Unis, pour déceler les cas d'autisme, on a utilisé des données des systèmes scolaires (seules ou avec des données cliniques) et des données provenant d'autres programmes gouvernementaux. Les Centers for Disease Control and Prevention ont mis sur pied un réseau de surveillance des TSA et d'autres déficiences du développement qui puise des données dans diverses sources ${ }^{21}$.

Les enfants de 8 ans atteints de TSA qui habitent dans l'un des 16 États constituant une partie de la zone de surveillance du réseau ont été recensés suivant une méthode en deux étapes. Dans la première étape, les enfants soupçonnés de présenter des TSA sont repérés par dépistage et par extraction de données issues de diverses sources, dans les dossiers médicaux et les dossiers scolaires. Dans la deuxième étape, les cliniciens attribuent une cote aux données extraites sur le comportement en vue de déterminer si l'enfant répond à la définition de cas de TSA. Les taux ont varié quelque peu d'un endroit à l'autre, mais le taux de prévalence moyen était en général de 6,6 pour 1000 enfants de 8 ans $^{22}$. Le réseau de surveillance a intégré un nombre considérable de fonctions relatives à l'assurance de la qualité afin d'obtenir des données de la plus grande qualité et des plus cohérentes. Newschaffer et al. ont utilisé une source nationale de données administratives (d'un organisme du ministère de l'Éducation des États-Unis responsable des programmes d'éducation spécialisée) pour dégager les tendances en matière de TSA entre 1992 et 2001. Cependant, les auteurs ont constaté des faiblesses dans ces données, plus particulièrement en ce qui concerne le classement spécifique des déficiences; il est probable que la prévalence de l'autisme calculée uniquement à partir des données sur l'éducation spécialisée soit sousestimée $^{23}$. En Californie, les personnes atteintes d'autisme (ou d'autres affections) sont admissibles à recevoir des services grâce au ministère responsable des services aux personnes atteintes d'un problème de développement (Department of Developmental Services). L'admissibilité est établie à partir du diagnostic posé et communiqué au Ministère par un professionnel de la santé compétent. Croen et $a{ }^{24}$ se sont servis de ces données pour estimer la prévalence de l'autisme. Ils se doutaient que la prévalence de 12,3 cas pour 10000 enfants qu'ils avaient obtenue pour les années 1987 à 1994 était inférieure à la valeur réelle, car environ 20 à $25 \%$ des enfants admissibles à recevoir des services n'étaient pas inscrits au programme ${ }^{24}$.

Au Canada, dans toutes les provinces et tous les territoires, des données administratives sont consignées sur l'hospitalisation et sur les consultations médicales, entre autres. En Nouvelle-Écosse, l'ajout d'une base de données sur les patients fréquentant une clinique externe de santé mentale a entraîné un accroissement d'environ 7 \% de la sensibilité des diagnostics de TSA, par rapport à la sensibilité calculée uniquement à partir des données sur l'hospitalisation et des données sur les demandes de remboursement des médecins. De même, l'exclusion des données sur les patients en clinique externe de santé mentale a permis d'augmenter la spécificité d'environ $6 \%$. Puisque relativement peu d'enfants ont été hospitalisés pour cause d'autisme ou présentaient un tel trouble au moment de l'hospitalisation (seulement $12 \%$ des vrais positifs avaient un code d'autisme provenant des données sur l'hospitalisation), la base de données sur l'hospitalisation, en tant que telle, ne s'est pas révélée adéquate pour le recensement des diagnostics d'autisme dans une population. Cependant, un diagnostic d'autisme dans la base de données sur l'hospitalisation était fort probablement correct. Bien que nous ayons considéré les codes de la CIM autres que ceux des TSA qui ont été utilisés, leur usage était trop peu généralisé pour nous permettre de proposer un algorithme qui pourrait réduire les taux de faux positifs ou de faux négatifs.

La recherche ou la surveillance de problèmes de santé à l'aide de bases de données administratives sur la santé présente des avantages sur d'autres méthodes de cueillette de données, car toutes les provinces et tous les territoires du Canada disposent de bases de données administratives sur la santé, lesquelles constituent la source d'un grand nombre de cas dans la population. Aussi, l'utilisation de ces bases de données coûte probablement moins cher que l'élaboration d'une nouvelle base de données. De plus, les personnes qui consignent les diagnostics dans les bases de données ne connaissent pas les hypothèses sous-jacentes relatives à l'exposition et à l'issue de l'exposition. Cependant, les bases de données administratives ont leurs faiblesses, plus particulièrement en ce qui concerne l'exactitude des diagnostics consignés à des fins de facturation (c'est le cas de la base de données sur la facturation des médecins au régime d'assurance-santé).

Étant donné la sensibilité maximale de 69 \% que nous avons mesurée, il est probable que si nous ne tenions compte que des données administratives sur la santé, nous obtiendrions des valeurs d'incidence et de prévalence inférieures à ce qu'elles devraient être en réalité, comme nous l'avons constaté dans cette étude. Il semble donc qu'il faudrait puiser dans des sources de données additionnelles pour améliorer le taux de détection des diagnostics de TSA à partir des bases de données existantes, car il est très peu probable qu'une seule source de données administratives fournisse le tableau complet des cas d'autisme au Canada. Malgré les difficultés que cela comporte, les autorités compétentes devraient se concerter pour acquérir des données normalisées, de diverses sources, en vue de mettre en place une surveillance passive continue des TSA au Canada. 


\section{Remerciements}

Cette étude a été réalisée grâce à la contribution financière de la fondation Cure Autism Now (maintenant appelée Autism Speaks). Les travaux de Linda Dodds ont été rendus possibles grâce à la Bourse d'études en recherche clinique de l'Université Dalhousie et à la Bourse de nouveau chercheur des Instituts de recherche en santé du Canada. Les auteurs tiennent à remercier les responsables du Reproductive Care Program of Nova Scotia (programme de soins en matière de reproduction de la Nouvelle-Écosse) et de l'unité de recherche sur la santé de la population de l'Université Dalhousie, qui ont offert l'accès à leurs données. Bien que l'étude soit en partie fondée sur les données de l'unité de recherche sur la santé de la population, les observations et les opinions exprimées dans le présent article sont celles des auteurs et ne reflètent pas nécessairement celles de l'unité.

\section{Références}

1. Bryson SE, Smith IM. Epidemiology of autism: prevalence, associated characteristics, and implications for research and service delivery. Ment Retard Dev Disabil Res Rev. 1998; 4:97-103.

2. Fombonne E. The prevalence of autism. JAMA. 2003;289:87-9.

3. Fombonne E. Epidemiology of autistic disorder and other pervasive developmental disorders. J Clin Psychiatry. 2005;66 Suppl 10:3-8.

4. Williams JG, Higgins JP, Brayne CE. Systematic review of prevalence studies of autism spectrum disorders. Arch Dis Child. 2006;91:8-15.

5. Filipek PA, Accardo PJ, Baranek GT, et al. The screening and diagnosis of autistic spectrum disorders. J Autism Dev Disord. 1999;29:439-84.

6. Bryson SE, Clark BS, Smith IM. First report of a Canadian epidemiological study of autistic syndromes. J Child Psychol Psychiatry. 1988; 29:433-45.
7. American Psychiatric Association. DSM IV diagnostic and statistical - manual. 4 ième éd., Washington (D.C.), American Psychiatric Association, 1994.

8. Ouellette-Kuntz H, Coo H, Yu CT, Chudley $\mathrm{AE}$, Noonan $\mathrm{A}$, Breitenbach $\mathrm{M}$, et al. Prevalence of pervasive developmental disorders in two Canadian provinces. J Appl Res Intellect Disabil. 2006;3:164-72.

9. Fombonne E, Zakarian R, Bennett A, Meng L, McLean-Heywood D. Pervasive developmental disorders in Montreal, Quebec, Canada: prevalence and links with immunizations. Pediatrics. 2006;118:e139-50.

10. To T, Dell S, Dick P, Cicutto L, Harris J, Tassoudji M, Duong-Hua M. Burden of childhood asthma, Toronto (Ontario), ICES, 2004.

11. Lix LM, Yogendran MS, Leslie WD, Shaw SY, Baumgartner R, Bowman C, et al. Using multiple data features improved the validity of osteoporosis case ascertainment from administrative databases. J Clin Epidemiol. 2008;61:1250-60.

12. Hux JE, Ivis F, Flintoft V, Bica A. Determination of prevalence and incidence using a validated administrative data algorithm. Diab Care. 2002;25:512-6.

13. Bearelly S, Mruthyunjaya P, Tzeng JP, Suner IJ, Shea AM, Lee JT, et al. Identification of patients with diabetic macular edema from claims data. Arch Ophthalmol. 2008;126:986-9.

14. Quan H, Li B, Saunders D, Parsons GA, Nilsson CI, Alibhai A, et al. Assessing validity of CIM-9CM and CIM-10 administrative data in recording clinical conditions in a unique dually coded database. Health Serv Res. 2008;43:1424-41.

15. Lord C, Rutter M, Le Couteur A. Autism diagnostic interview-revised: a revised version of a diagnostic interview for caregivers of individuals with possible pervasive developmental disorders. J Autism Dev Disord. 1994;24:659-85.

16. Lord C, Risi S, Lambrecht L, Cook EH, Leventhal EL, DiLavore PC, et al. The autism diagnostic observation schedulegeneric: a standard measure of social and communication deficits associated with the spectrum of autism. J Autism Dev Disord. 2000;30:205-23.

17. American Psychiatric Association. Diagnostic and statistical manual. 4 ième éd. Washington (D.C.), American Psychiatric Association, 2000.

18. Filipek PA, Accardo PJ, Baranek GT, Cook EH, Dawson G, Gordon B, et al. The screening and diagnosis of autistic spectrum disorders. J Autism Dev Disord. 1999;29:439-84.

19. Filipek PA, Accardo PJ, Ashwal S, Baranek GT, Cook EH, Dawson G, et al. Practice parameter: screening and diagnosis of autism: report of the quality standards subcommittee of the American academy of neurology and the child neurology society. Neurology. 2000;55:468-79.

20. Ash A, Shwartz M. R ${ }^{2}$ : A useful measure of model performance when predicting a dichotomous outcome. Stat Med. 1999; 18:375-84.

21. Rice CE, Baio J, Van Naarden Braun K, Doernberg N, Meaney FJ, Kirby RS. A public health collaboration for the surveillance of autism spectrum disorders. Paediatr Perinat Epidemiol. 2007;21:179-90.

22. Autism and Developmental Disabilities Monitoring Network Surveillance Year 2002 Principal Investigators; Centers for Disease Control and Prevention. Prevalence of autism spectrum disorders--autism and developmental disabilities monitoring network, 14 sites, United States, 2002. MMWR Surveill Summ. 2007 Feb 9; 56(1):12-28.

23. Newschaffer CJ, Falb MD, Gurney JG. National autism prevalence trends from United States special education data. Pediatrics. 2005;115:e277-82.

24. Croen LA, Grether JK, Selvin S. Descriptive epidemiology of autism in a California population: who is at risk? J Autism Dev Disord. 2002;32:217-24. 